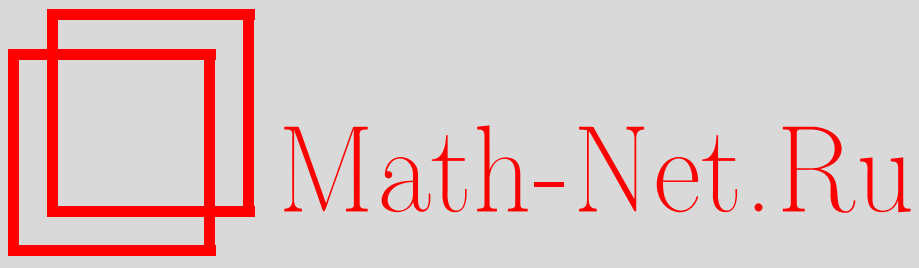

В. С. Лубенцова, А. В. Ефремов, Решение задачи определения вместимости контейнерного терминала с использованием модели "гибели и рождения", Вестн. Сам. гос. техн. ун-та. Сер. Физ.-мат. науки, 2005, выпуск 38, 155-158

DOI: https://doi.org/10.14498/vsgtu393

Использование Общероссийского математического портала Math-Net.Ru подразумевает, что вы прочитали и согласны с пользовательским соглашением

http://www . mathnet.ru/rus/agreement

Параметры загрузки:

IP : 54.198 .67 .100

26 апреля 2023 г., 05:04:31

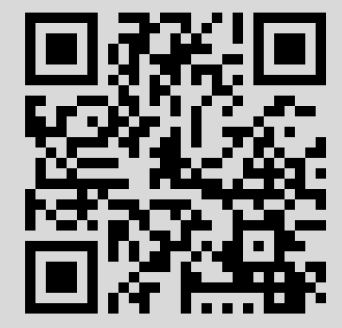




\section{РЕШЕНИЕ ЗАДАЧИ ОПРЕДЕЛЕНИЯ ВМЕСТИМОСТИ КОНТЕЙНЕРНОГО ТЕРМИНАЛА С ИСПОЛЬЗОВАНИЕМ МОДЕЛИ «ГИБЕЛИ И РОЖДЕНИЯ»}

Проведен расчет вместимости зоны хранения контейнерного терминала с использованием модели «гибели и рождения» теории массового обслуживания. Построены зависимости интегральной функции распределения от числа хранящихся на складе контейнеров, позволяющие определить вместимость зоны хранения с заданной вероятностью.

Для определения такой важной характеристики как вместимость грузообрабатывающего предприятия (склад, терминал, логистический центр) используются разные подходы: вероятностно-статистические методы, имитационное моделирование, математический аппарат систем массового обслуживания.

В данной работе проведен анализ такой системы с использованием модели «гибели и рождения» теории массового обслуживания $[1,2]$. Контейнерный терминал рассматривается как открытая система массового обслуживания (СМО) с ожиданием (рис. 1).

Терминал рассматривается как многоканальная СМО, причем под блоком обслуживания принимается место размещения некоторой транспортной партии, в частности контейнеров. Состояние СМО с ожиданием определяется числом контейнеров, находящихся в зоне хранения (целые неотрицательные числа). Процесс перехода из состояния в состояние является марковским процессом. При этом следует учитывать, что изменение состояния имеет две причины: 1) поступление новых контей-

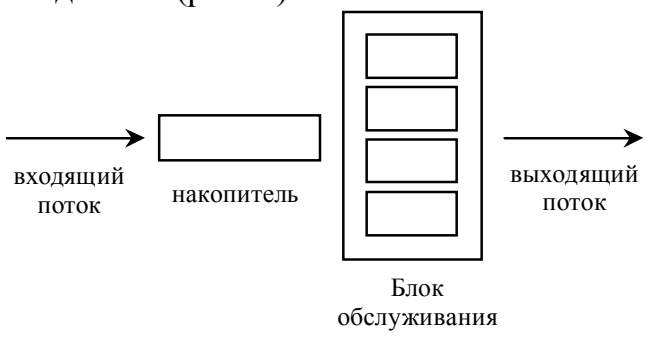

Р и с. 1. Схема открытой СМО с ожиданием неров из входящего потока; 2) уход обслуживания контейнеров из зоны хранения.

Изменение состояния по первой причине обладает марковским свойством, поскольку входящий поток является простейшим [3]. Поступление контейнеров в зону хранения на любом отрезке времени не зависит от того, сколько контейнеров поступило до начального момента этого отрезка. С другой стороны, если в какой то момент времени контейнер покидает зону хранения, то вероятность окончания обслуживания не зависит от того, сколько длилось хранение. Таким образом, изменение состояния СМО по второй причине также обладает марковским свойством. В связи с этим можно определить вероятности переходов. Переход СМО из состояния $q$ в состояние $q+1$ за время $t$ связан с поступлением одной партии груза (одного контейнера) в систему. Эта вероятность определяется законом Пуассона:

$$
P_{k}(t)=\frac{(\lambda t)^{k}}{k !} e^{-\lambda t}
$$

и при $k=1$ равна

$$
P_{1}(t)=\lambda t e^{-\lambda t},
$$

где $\lambda$ - интенсивность входящего потока.

Длительность обслуживания распределена по экспоненциальному закону с интенсивностью обслуживания $\mu$ :

$$
P\left(t_{\text {обсл }}>t\right)=\int_{t}^{\infty} \mu e^{-\mu t} d t=e^{-\mu t} .
$$

Если $k$ - число занятых мест зоны хранения в $q$-том состоянии, а $n-$ общее число мест в зоне хранения, то число занятых мест зоны хранения при $q \leq n$ равно $q$ и $n$ при $q \geq n$.

Вероятность того, что за время $t$ ни одно из занятых мест не освободилось, равна

$$
P_{0}(t)=\left(e^{-\mu t}\right)^{k} .
$$

Следовательно, для $q \leq n$ имеем

$$
P_{q, q+1}(t)=\lambda t e^{-\lambda t}\left(e^{-\mu t}\right)^{q} .
$$

Учитывая известное разложение функции $e^{x}$, имеем 
Тогда

$$
\begin{gathered}
e^{-\lambda t}=1-\lambda t+0(t), \\
\left(e^{-\mu t}\right)^{q}=e^{-q \mu t}=1-q \mu t+0(t) .
\end{gathered}
$$

Аналогично при $q \geq n$

$$
P_{q, q+1}(t)=\lambda t(1-\lambda t+0(t))(1-q \mu t+0(t))=\lambda t+0(t) .
$$

$$
P_{q, q+1}(t)=\lambda t(1-\lambda t+0(t))(1-n \mu t+0(t))=\lambda t+0(t) .
$$

Переход $q \rightarrow q-1$ связан с отсутствием поступлений контейнеров в зону хранения и уходом из зоны хранения одного контейнера.

При $q \leq n$ имеем

$$
P_{q, q-1}(t)=e^{-\lambda t}\left(e^{-\mu t}\right)^{q-1} C_{q}^{1} \mu t e^{-\mu t}=q \mu t+0(t) .
$$

Аналогично при $q \geq n$

$$
P_{q, q-1}(t)=n \mu t+0(t) .
$$

Таким образом, функционирование рассматриваемой СМО описывается процессом «гибели и рождения» [4]. При этом

$$
\lambda_{k}=\lambda, \mu_{k}=\left\{\begin{array}{l}
k \mu, \text { при } k \leq n, \\
n \mu, \text { при } k \geq n .
\end{array}\right.
$$

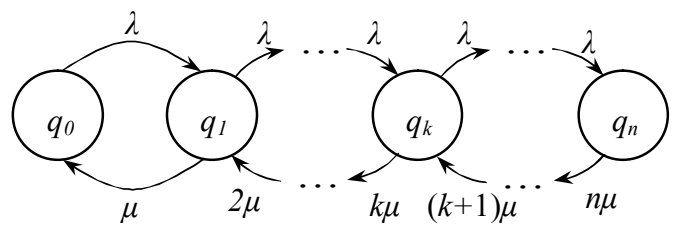

Р и с. 2. Граф состояний открытой СМО: $q_{0}$ - зона хранения терминала пуста; $q_{1}-$ в зоне хранения находится один контейнер; $q_{2}-$ в зоне хранения находится два контейнера;

$q_{n}-$ в зоне хранения находятся $n$ контейнеров (свободных мест нет)

Граф состояний такой системы имеет вид, представленный на рис. 2.

В соответствии с теорией массового обслуживания финальные вероятности в процессе «гибели и рождения» находятся из соотношений

$$
\left\{\begin{array}{l}
P_{0}=\frac{1}{1+\sum_{j=0}^{\infty} \frac{\lambda_{0} \lambda_{1} \ldots \lambda_{j-1}}{\mu_{1} \mu_{2} \ldots \mu_{j}},} \\
P_{j}=P_{0} \frac{\lambda_{0} \lambda_{1} \ldots \lambda_{j-1}}{\mu_{1} \mu_{2} \ldots \mu_{j}}, j \geq 1 .
\end{array}\right.
$$

После подстановки (12) в (13) получим финальные вероятности при $k \leq n$

при $k \geq n$

$$
P\left(q_{k}\right)=\frac{\lambda}{k \mu} \cdot \frac{\lambda}{(k-1) \mu} \ldots \frac{\lambda}{\mu} P_{0}=\frac{\lambda^{k}}{\mu^{k} k !} P_{0},
$$

$$
P\left(q_{n}\right)=\underbrace{\frac{\lambda}{n \mu} \ldots \frac{\lambda}{n \mu}}_{k-n} \frac{\lambda}{n \mu} \frac{\lambda}{(n-1) \mu} \ldots \frac{\lambda}{\mu} P_{0}=\frac{\lambda^{k}}{\mu^{k} n ! n^{k-n}} P_{0} .
$$

Так как процесс обслуживания заявок в рассматриваемом случае представляет собой пребывание груза в зоне хранения в течение среднего времени $\tau_{x p}$, то интенсивность выходящего потока $\mu=\frac{1}{\overline{\tau_{x p}}}$. Вероятность того, что в зоне хранения терминала будет находиться $k$ партий груза $(k<n)$, определяется соотношением

$$
P\left(q_{k}\right)=\frac{\lambda^{k} \bar{\tau}_{x p}^{k}}{k !} P\left(q_{0}\right) .
$$

Вероятность того, что зона хранения груза будет заполнена полностью, имеет вид

$$
P\left(q_{n}\right)=\frac{\lambda^{n} \bar{\tau}_{x p}^{n}}{n !} P\left(q_{0}\right) .
$$

Вероятность того, что зона хранения грузов заполнена не полностью и вновь прибывшая партия может быть обслужена, принята равной 


$$
P(k<n)=1-\frac{\lambda^{k} \bar{\tau}_{x p}^{k}}{k !} P\left(q_{0}\right)
$$

Так как $\sum_{k=1}^{n} P\left(q_{k}\right)=1$, то

$$
P\left(q_{0}\right)=\left(1+\lambda \bar{\tau}_{x p}+\frac{1}{2 !} \lambda^{2} \bar{\tau}_{x p}^{2}+\frac{1}{3 !} \lambda^{3} \bar{\tau}_{x p}^{3}+\ldots+\frac{1}{n !} \lambda^{n} \bar{\tau}_{x p}^{n}\right)^{-1}=\frac{1}{1+\sum_{k=1}^{n} \frac{I^{k}}{k !}} .
$$

Здесь $I=\lambda \bar{\tau}_{x p}$ - средний запас грузов в зоне хранения терминала.

Стоящее в знаменателе (19) выражение при достаточно большом $n$ представляет собой ряд Маклорена для функции $e^{I}$, поэтому приближенно можно считать

$$
\begin{gathered}
P\left(q_{0}\right)=\frac{1}{e^{I}}, \\
P\left(q_{k}\right)=\frac{I^{k}}{k !} e^{-I}, \\
P(k<n)=1-\frac{I^{k}}{k !} e^{-I} .
\end{gathered}
$$

Для контейнерных терминалов интенсивность входящего потока определяется достаточно большим числом - количеством поступающих терминалов $\lambda=150 \div 300$, и формула Пуассона (21) аппроксимируется формулой нормального распределения

$$
P\left(q_{k}\right)=\frac{1}{\sqrt{2 \pi I}} e^{-\frac{(k-I)^{2}}{2 I}} .
$$

По этой формуле были построены графики распределений количеств фактически хранящихся контейнеров $k$ при разной средней вместимости зоны хранения терминала (рис. 3 ). На рис. 4 представлены зависимости интегральной функции распределения от числа хранящихся контейнеров при разной средней загрузке терминала:

$$
F\left(q_{k}\right)=\sum_{k=1}^{n} \frac{1}{\sqrt{2 \pi I}} e^{-\frac{(k-I)^{2}}{2 I}} .
$$

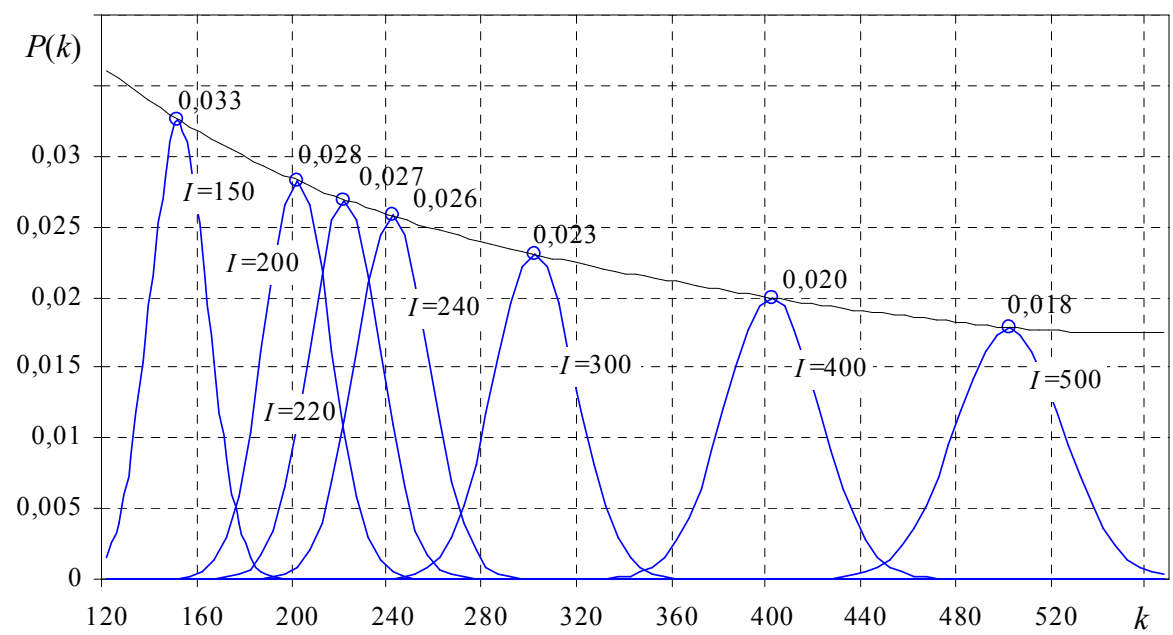

Р и с. 3. Графики распределений количеств фактически хранящихся контейнеров при разной средней вместимости площадки 


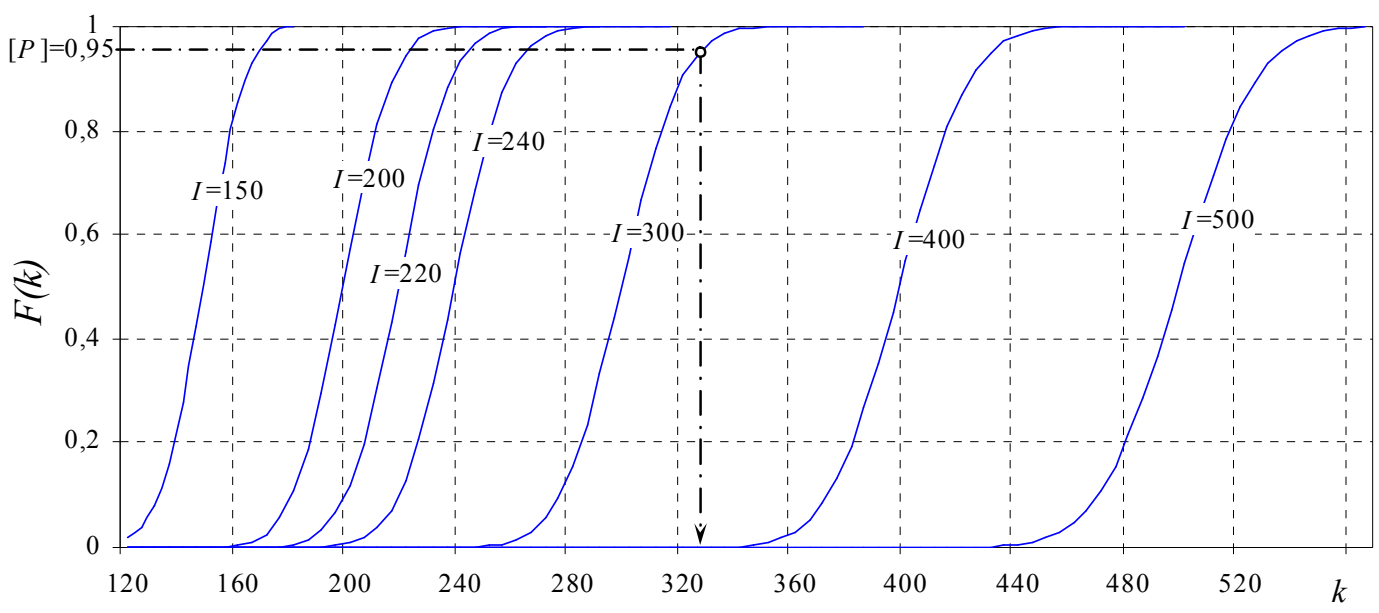

Р и с. 4. Зависимость интегральной функции распределения $F(k)$ от числа фактически хранящихся контейнеров при разной средней вместимости площадки

Построенные графики дают возможность определить потребную вместимость терминала с заданной доверительной вероятностью. Из графиков видно, что средний запас грузов на складе соответствует наиболее вероятному запасу, однако он может быть превышен. С увеличением размера терминала абсолютная величина возможного отклонения действительного количества хранящегося груза от среднего значения увеличивается. При доверительной вероятности $[p]=0,95$ относительное отклонение не превышает $15 \div 20 \%$.

\section{БИБЛИОГРАФИЧЕСКИЙ СПИСОК}

1. Маликов О. Б. Деловая логистика. СПб: Политехника, 2003. 223 с.

2. Маликов О. Б. Склады гибких производств. Л.: Машиностроение, 1986. 183 с.

3. Вентиель Е. С., Овчаров Л. А. Теория вероятности и ее инженерные приложения. М.: Наука, 1991. 383 с.

4. Тихоненко О. М. Модели массового обслуживания в информационных системах. Минск: Техпринт, 2003. 327 с.

Поступила 9.02.2005 2.

УДК $57.01+577.4$

\section{Л. С. Бекасов}

\section{О СТРАТЕГИЯХ МОДЕЛИРОВАНИЯ ГЕНЕТИЧЕСКИХ СТРУКТУР}

Представлены основные сведения из начал генетики, касающиеся построения дезоксирибонуклеиновой кислоты (ДНК) и синтеза белков. Описаны основные особенности построения этой сверхсложной системы, касающиеся её формы и сущности её элементарных компонент - нуклеотидов. Предложены некоторые подходы формализачии нуклеотидов и их характера связей в биоструктуpax.

Элементарной единицей биологических систем является органическая клетка. Внутри таких клеток у эукароиот (растения, животные, люди) содержится молекула дезоксирибонуклеиновой кислоты (ДНК). ДНК, как считают биологи, хранит в себе всю информацию о строении того организма, которому она принадлежит $[1,2]$. Конструктивно эта молекула представляет собой «веревочную лестницу», скрученную в спираль. Ступеньки этой лестницы образуются за счет химических структур, четырех основных органических соединений - нуклеотид, именуемых как аденин (A), тимин (T), гуанин $(\mathrm{G})$ и цитозин $(\mathrm{C})$. Эти конструкции в ДНК соединяются строго попарно на комплементарной основе (аденин - тимин, гуанин - цитозин). Особенность в их соединении определяется тем, что оно реализуется только при помощи ато - 